\title{
COMMISSIONING RESULTS OF THE KEKB AND PEP-II B-FACTORIES*
}

\author{
J. T. Seeman $^{\#}$, MS 18, SLAC, Stanford, CA 94309
}

\section{KEKB Abstract}

KEKB [1-6] is a two ring asymmetric electron-positron collider at $3.5 \times 8 \mathrm{GeV}$ being built in the TRISTAN tunnel at the Japan National Laboratory for High Energy Physics KEK in Tsukuba. The five year KEKB construction project was approved by the Japanese government in April 1994. The parameters are listed in Section 1 and the overall layout of KEKB shown in Fig. 1. The two beams will collide in the Tsukuba experimental hall where the BELLE detector will be located. The salient features of KEKB are side-by-side rings, +/- $11 \mathrm{mrad}$ crossing angles at the collision point with non-radiating straight incoming beams, new RF cavities: copper cavities with high stored energy (ARES) and superconducting cavities both designed to reduce the strength of the bunch-by-bunch feedback systems, many bunches (5000), high stored beam currents (2.6 A x $1.1 \mathrm{~A})$, superconducting IR quadrupoles, novel $2.5 \pi$ lattice design for flexibility, and an upgraded linac for full energy injection of both beams with new transport lines.

Beam commissioning started for the KEKB High Energy Ring HER in December 1998 and January 1999 for the Low Energy Ring LER. The present peak current in the LER is $380 \mathrm{~mA}$ and the HER $420 \mathrm{~mA}$. A luminosity

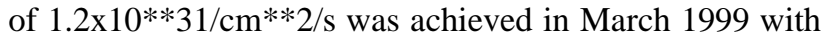
200 bunches with $13 \mathrm{~mA}$ x $65 \mathrm{~mA}$.

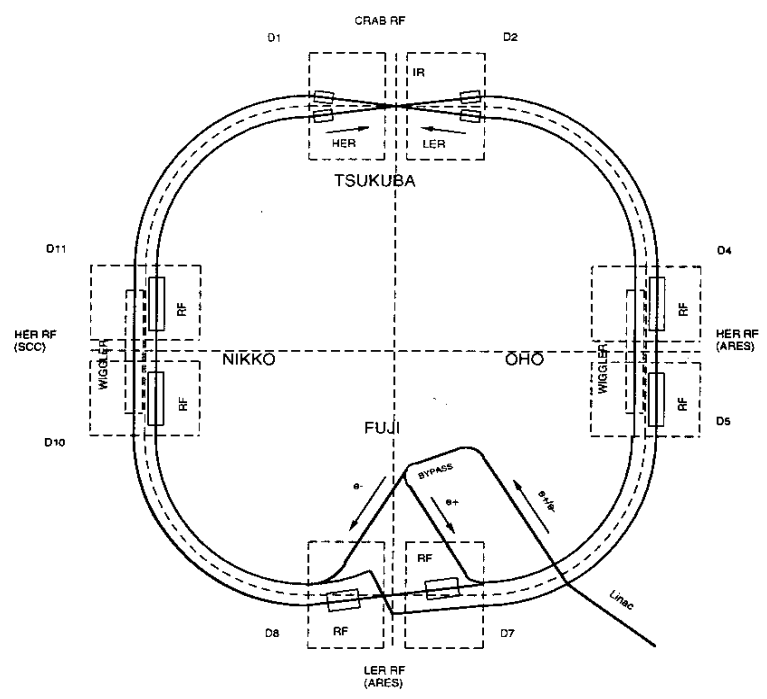

Figure 1: KEKB B-Factory Overview

*Work supported in Department of Energy contract number DEAC03-76SF00515.

\# Email: SEEMAN@slac.stanford.edu
PEP-II Abstract

PEP-II [7-12] is a two ring asymmetric electron-positron collider at $3.1 \times 9.0 \mathrm{GeV}$ being built in the PEP tunnel at the Stanford Linear Accelerator Center SLAC. The five year PEP-II construction project was approved by the Department of Energy of the United States government in January 1994. A collaboration of SLAC, Lawrence Berkeley National Laboratory LBNL, and Lawrence Livermore National Laboratory LLNL designed and constructed PEP-II. The parameters are listed in Section 3 and the overall layout of PEP-II is shown in Fig. 2. The beams will collide in the IR2 hall of the PEP tunnel where the BABAR detector will be located. The salient features of PEP-II are LER-above-the-HER, head-on collisions but with dipoles bends near the collision point, new copper RF cavities, strong bunch-by-bunch feedback systems, many bunches (1658), high stored charges (2.1 A x 0.75 A), permanent magnet IR quadrupoles and dipoles, and use of the existing SLAC linac as an injector but with new transport lines.

PEP-II HER commissioning started in June 1997 and LER commissioning in July 1998. The present maximum current in the HER is $750 \mathrm{~mA}$ in 1658 bunches which is the design value. The LER has stored $1171 \mathrm{~mA}$, which is believed to be a world record for a positron ring. A luminosity of $5.2 \times 10 * * 32 / \mathrm{cm}^{* * 2} 2 / \mathrm{s}$ was achieved in February 1999 with 786 bunches with $354 \mathrm{~mA}$ x $680 \mathrm{~mA}$ (HER x LER). Bunch trains of 1, 11, 291, 522, 786, 1048, and 1571 have been collided. An average luminosity of $2 \times 10 * * 32 / \mathrm{cm}^{*} * 2 / \mathrm{s}$ was maintained over 72 hours.

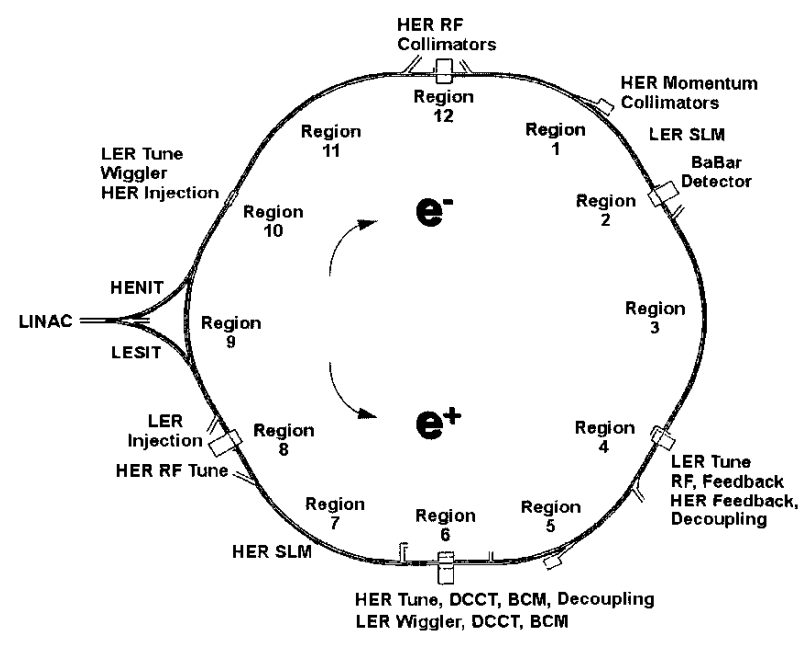

Figure 2: PEP-II B-Factory Overview 


\section{KEKB DESIGN PARAMETERS}

\begin{tabular}{|c|c|c|}
\hline Parameter & $\underline{\mathrm{LER}}$ & $\underline{\mathrm{HER}}$ \\
\hline Beam energy (GeV) & 3.5 & 8.0 \\
\hline CM energy $(\mathrm{GeV})$ & \multicolumn{2}{|c|}{10.58} \\
\hline Particle type & $\mathrm{e}+$ & e- \\
\hline Circumference (m) & \multicolumn{2}{|c|}{3016.3} \\
\hline Crossing angle (mrad) & \multicolumn{2}{|c|}{$+/-11.0$} \\
\hline Luminosity & \multicolumn{2}{|c|}{$10^{34} \mathrm{~cm}^{-2} \mathrm{sec}^{-1}$} \\
\hline Beam-beam tune shift (y) & \multicolumn{2}{|c|}{0.052} \\
\hline Beam-beam tune shift (x) & \multicolumn{2}{|c|}{0.039} \\
\hline$\beta y^{*} / \beta x^{*}(\mathrm{~cm} / \mathrm{cm})$ & $1 . / 33$. & $1 . / 33$. \\
\hline Optimum coupling (\%) & 2.0 & 2.0 \\
\hline Emittance (nm-rad) (y/x) & $0.36 / 25$ & $0.36 / 25$ \\
\hline IP $\mathrm{rms}$ beam $\sigma_{\mathrm{y}} / \sigma_{\mathrm{x}}(\mathrm{mm})$ & $1.9 / 90$ & $1.9 / 90$ \\
\hline Number of bunches & 5000 & 5000 \\
\hline Particles per bunch & $3.3 \times 10^{10}$ & $1.4 \times 10^{10}$ \\
\hline Bunch spacing (m) & 0.59 & 0.59 \\
\hline Bunch length (mm) & 4.0 & 4.0 \\
\hline Damping times $(\mathrm{ms})(\mathrm{x} / \mathrm{z})$ & 23 & 23 \\
\hline$\Delta \mathrm{E} /$ turn $(\mathrm{MeV})$ & 1.5 & 3.5 \\
\hline Radiation power (MW) & 4.0 & 3.8 \\
\hline HOM power (MW) & 0.57 & 0.14 \\
\hline Total beam power (MW) & 4.5 & 4.0 \\
\hline Total current $(\mathrm{A})$ & 2.6 & 1.1 \\
\hline RF frequency (MHz) & 508.9 & 508.9 \\
\hline Harmonic number & 5120 & 5120 \\
\hline Ion clearing gap (buckets) & --- & 120 \\
\hline RF voltage (MV) & 8 & 20 \\
\hline Rel. energy spread $\left(10^{-3}\right)$ & 0.71 & 0.67 \\
\hline Synchrotron tune & 0.012 & 0.011 \\
\hline Betatron tune $\left(v_{\mathrm{x}} / \mathrm{v}_{\mathrm{y}}\right)$ & $45.71 / 44.49$ & $44.51 / 42.29$ \\
\hline Linac injection rate $(\mathrm{Hz})$ & 50 & 50 \\
\hline Full injection time (min) & $13-14$ & 3 \\
\hline Detector solenoid field (T) & & \\
\hline
\end{tabular}

\section{KEKB MILESTONES}

$\begin{array}{lll}\underline{\text { Date }} & & \text { Milestone or Commissioning } \\ 1994 & \text { April } & \text { Start of KEKB construction } \\ 1995 \text { December } & \text { Start removing TRISTAN } \\ 1995 \text { July } & \text { Bids out for LER construction } \\ 1996 \text { May } & \text { Bids out for HER construction } \\ 1996 \text { July-Nov. } & \text { Beam tests in Accum. Ring } \\ 1996 \text { December } & \text { Start bypass tunnel constr. } \\ 1997 \text { February } & \text { Start magnet installation } \\ 1997 \text { October } & \text { Complete new bypass tunnel } \\ 1998 \text { May } & \text { Commission fully upgraded } \\ 1998 \text { Nov. 30 } & \text { KEKB installation complete } \\ 1998 \text { Dec. 13 } & \text { HER beam first stored } \\ 1999 \text { Jan. 14 } & \text { LER beam first stored } \\ 1999 \text { Jan. 26 } & \text { First beam-beam collisions } \\ 1999 \text { March 26 } & \text { Luminosity of 1.2x10**31 } \\ 1999 \text { May } & \text { BELLE detector to be installed } \\ 2000 & \text { Nine month run planned }\end{array}$

\section{PEP-II DESIGN PARAMETERS}

\begin{tabular}{|c|c|c|}
\hline$\underline{\text { Parameter }}$ & $\underline{\text { LER }}$ & $\underline{\mathrm{HER}}$ \\
\hline Beam energy (GeV) & 3.1 & 9.0 \\
\hline CM energy $(\mathrm{GeV})$ & \multicolumn{2}{|c|}{10.58} \\
\hline Particle type & $\mathrm{e}+$ & e- \\
\hline Circumference (m) & \multicolumn{2}{|c|}{2199.318} \\
\hline Crossing angle (mrad) & \multicolumn{2}{|c|}{0.0} \\
\hline Luminosity & \multicolumn{2}{|c|}{$3 \times 10^{33} \mathrm{~cm}^{-2} \mathrm{sec}^{-1}$} \\
\hline Beam-beam tune shift (y) & \multicolumn{2}{|c|}{0.03} \\
\hline Beam-beam tune shift (x) & \multicolumn{2}{|c|}{0.03} \\
\hline$\beta y^{*} / \beta x^{*}(\mathrm{~cm} / \mathrm{cm})$ & $1.5 / 50$ & $1.5 / 50$ \\
\hline Optimum coupling (\%) & 3.0 & 3.0 \\
\hline Emittance (nm-rad) (y/x) & $1.5 / 49$ & $1.5 / 49$ \\
\hline IP $\mathrm{rms}$ beam $\sigma_{\mathrm{y}} / \sigma_{\mathrm{x}}(\mathrm{mm})$ & $4.7 / 157$ & 4.7/157. \\
\hline Number of bunches & 1658 & 1658 \\
\hline Particles per bunch & \multicolumn{2}{|c|}{$6.0 \times 10^{10} 2.8 \times 10^{10}$} \\
\hline Bunch spacing (m) & 1.26 & 1.26 \\
\hline Bunch length (mm) & 10.0 & 11.0 \\
\hline Damping times $(\mathrm{ms})(\mathrm{x} / \mathrm{z})$ & $62.5 / 30$ & $37 . / 18.3$ \\
\hline$\Delta \mathrm{E} /$ turn $(\mathrm{MeV})$ & 0.75 & 3.6 \\
\hline Radiation power (MW) & 1.62 & 3.58 \\
\hline HOM power (MW) & 0.23 & 0.15 \\
\hline Total beam power (MW) & 1.85 & 3.73 \\
\hline Total current (A) & 2.16 & 0.75 \\
\hline RF frequency (MHz) & 476. & 476. \\
\hline Harmonic number & 3492 & 3492 \\
\hline Ion clearing gap (buckets) & --- & 176 \\
\hline RF voltage (MV) & 5.1 & 14.0 \\
\hline Gap voltage/cavity (MV) & 0.85 & 0.7 \\
\hline Rel. energy spread $\left(10^{-3}\right)$ & 0.77 & 0.61 \\
\hline Synchrotron tune & 0.0334 & 0.0449 \\
\hline Betatron tune $\left(v_{\mathrm{x}} / \mathrm{v}_{\mathrm{y}}\right)$ & \multicolumn{2}{|c|}{$38.57 / 36.6424 .62 / 23.64$} \\
\hline Linac injection rate $(\mathrm{Hz})$ & 60 & 120 \\
\hline Detector solenoid field $(\mathrm{T})$ & 1.5 & \\
\hline
\end{tabular}

\section{PEP-II MILESTONES}

$\underline{\text { Date }}$

1994 January

1995 October

1997 April

1997 June

1997 June 16

1997 June

1998 January

1998 July 10

1998 July 16

1998 July 23

1998 Dec. 8

1998 Feb. 8

1999 May

2000
Milestone or Commissioning

Start of PEP-II Construction

e- injection transport studies

HER injection studies

HER installation complete

HER beam first stored

e+ injection transport studies

LER injection (part turn)

LER construction complete

LER beam first stored

First collisions

$8 \times 10 * * 31$ luminosity

$5.2 \times 10 * * 32$ luminosity

BABAR detector to be installed

Ten month run planned 


\begin{tabular}{lll}
\multicolumn{4}{c}{ KEKB COMMISSIONING RESULTS } \\
\multicolumn{1}{c}{ (as of 3/30/1999) } & LER & HER \\
Beam energy (GeV) & 3.5 & 8.0 \\
Maximum total current (mA) & 380 & 420 \\
Single bunch current (mA) & 2.3 & 4.0 \\
Number of bunches & 1024 & 640 \\
$\beta y^{*}(\mathrm{~cm})$ & 1.0 & 1.0 \\
$\beta x^{*}(\mathrm{~cm})$ & 100 & 100 \\
Bunch length (mm) & 6.4 & 5.6 \\
Injection rate (Hz) & 50 & 25 \\
RF voltage & 4.0 & 9.0 \\
Number of ARES RF cavities & 12 & 6 \\
Number of SC cavities & 0 & 4 \\
vx & 45.57 & 44.26 \\
vy & 44.62 & 42.21 \\
Max. injection efficiency (\%) & 70 & $80-100$ \\
Injection charge (nC) & 0.18 & 1.0 \\
Beam lifetime in collision (min) & 40 & 200 \\
Bunches collided (max) & \multicolumn{2}{c}{$200 \times 200$} \\
Collided currents (mA) & 65 & 13 \\
Maximum luminosity (1/cm $/ \mathrm{s})$ & $1.2 \times 10^{* * 31}$ \\
IP beam sizes (microns) (x,y) & \multicolumn{2}{c}{$197 / 5.6$} \\
Integrated current (A-hr) & 28 & 20
\end{tabular}

\section{PEP-II COMMISSIONING RESULTS}

\begin{tabular}{|c|c|c|}
\hline (as of 3/30/1999) & LER & HER \\
\hline Beam energy $(\mathrm{GeV})$ & 3.1 & 9.0 \\
\hline Maximum total current (mA) & 1171 & 750 \\
\hline Single bunch current (mA) & 7.0 & 12.0 \\
\hline Number of bunches & 1658 & 1658 \\
\hline$\beta y *(\mathrm{~cm})$ & 1.4 & 1.3 \\
\hline$\beta \mathrm{x}^{*}(\mathrm{~cm})$ & 50 & 50 \\
\hline Bunch length (mm) & --- & 12. \\
\hline Injection rate $(\mathrm{Hz})$ & 10 & 10 \\
\hline RF voltage (max) (MV) & 3.2 & 14 \\
\hline Number of cavities & 4 & 20 \\
\hline$v \mathrm{x}$ & 38.63 & 24.58 \\
\hline vy & 36.59 & 23.62 \\
\hline Bunch ion gap (\%) & 10 & 10 \\
\hline Max. injection efficiency (\%) & $90-100$ & $95-100$ \\
\hline Injection charge $(\max )(\mathrm{nC})$ & 3 & 3 \\
\hline Injection rate at $10 \mathrm{~Hz}(\mathrm{~mA} / \mathrm{sec})$ & 2.1 & 2.2 \\
\hline Beam lifetime at $300 \mathrm{~mA}(\mathrm{~min})$ & 150 & 420 \\
\hline Bunches collided (max) & \multicolumn{2}{|c|}{$1571 \times 1571$} \\
\hline Collided currents (max) (mA) & 1007 & 354 \\
\hline Luminosity $(\max )\left(1 / \mathrm{cm}^{2} / \mathrm{s}\right)$ & \multicolumn{2}{|c|}{$5.2 \times 10^{32}$} \\
\hline Current at max luminosity (mA) & 680 & 354 \\
\hline IP beam sizes (microns) $(\mathrm{x}, \mathrm{y})$ & \multicolumn{2}{|c|}{$160 / 6.1$} \\
\hline Integrated current (A-hr) & 153 & 115 \\
\hline
\end{tabular}

\section{KEKB COMMISSIONING ISSUES}

The magnetic lattices for KEKB have noninterleaved $2.5 \pi$ phase advance cells with local chromaticity correction near the interaction point. The measured lattice functions are close to the design and the IP vertical beta functions are $1.0 \mathrm{~cm}$ which is a world record level for an e+ecollider.

The KEKB LER uses ARES cavities. The ARES system has a HOM-damped copper accelerating cavity coupled to a high-Q energy storage cavity via a coupling cavity. The HER will use a combination of ARES and superconducting (SC) cavities. The SC cavities are single cells with room temperature beam line ferrite HOM absorbers. The KEKB RF systems worked well during commissioning. The measured synchrotron frequencies are correct. The superconducting cavities have had very few trips over the first few months. No longitudinal instabilities have been seen even without feedback.

The vacuum chambers for both rings of KEKB are extruded copper with Helicoflex seals. The HER chambers are racetrack shaped and have distributed NEG pumping. The LER chambers are circular to reduce the resistive wall instability and have NEG cartridges every meter. During commissioning the vacuum systems started with somewhat higher pressures than expected but with beam scrubbing the gas desorption rate and resulting pressures have quickly approached values expected at this commissioning phase. See Fig. 3.

The existing KEK linac has been upgraded to inject at full energy into KEKB. The upgrade involved adding more linac accelerating sections, newly designed SLED energy doublers, higher power klystrons, improved diagnostics, new positron target, and a new gun. The positron production per pulse has increased a factor of twenty. New transport lines were also built. Long term beam stability issues are under study for the injection complex.

Transversely, the beams are stable with bunch-bybunch feedback. Without feedback, an instability starts in the LER at about $100 \mathrm{~mA}$ for bunch spacings less that 8ns. The cause is under study. Instability in the HER appears as centroid motion at the end of a long bunch train at currents of about $190 \mathrm{~mA}$. In both rings growth times approach a few milliseconds at high currents.

In KEKB the LER and HER beams are brought into collision horizontally with $+/-11 \mathrm{mrad}$ crossing angles. The first quadrupoles QCS which are superconducting including skew quadrupole and $\mathrm{x}-\mathrm{y}$ dipole windings have been fully tested. Farther away, several special iron septum quadrupoles are needed in both rings to complete the IP doublet optics and to allow the two beams to separate. The compensation of the detector solenoid done by a pair of reverse solenoids near the collision point and with small trim skew quadrupoles in the interaction region has also been tested.

KEKB has had about four days of colliding beam experiments. The beams were first steered into collision using position monitors. Then, beam-beam scans are used to center the beams and measure their sizes. The scans were done magnetically in the vertical plane and longitudinally with the LER RF phase for horizontal 
scans. See Fig. 4. Most studies have been with single bunches but 200 bunches have been collided. The vertical and horizontal beam sizes are near the design values. A slow orbit drift of unknown origin affects IR steering.

Backgrounds near the IP have been measured by the BEAST detector. The backgrounds are high at this early phase but collimator and beam steering are starting to reduce the levels.

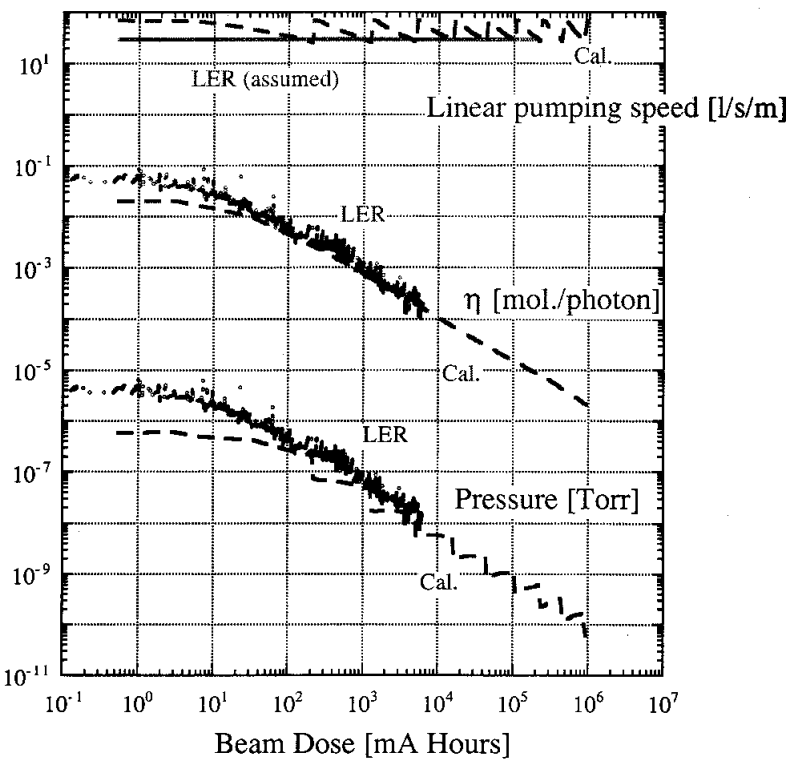

Figure 3: KEKB vacuum pressure and desorption versus beam dose. Scrubbing is following expectations (- - -).

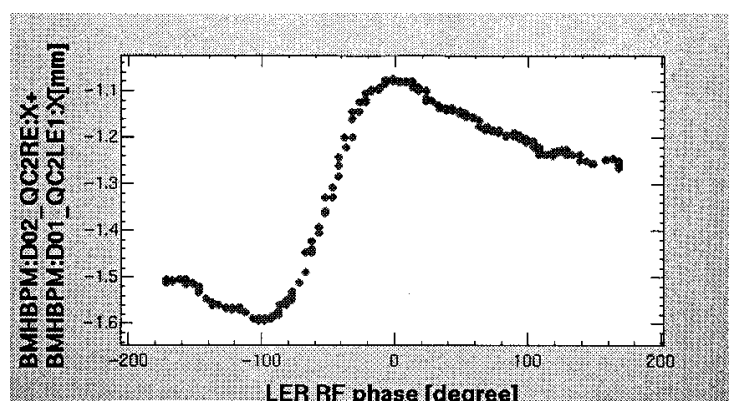

Figure 4: KEKB horizontal beam-beam deflection scan.

\section{PEP-II COMMISSIONING ISSUES}

The PEP-II magnetic lattices for the HER and the LER are significantly different. The HER has a 60 degree per cell lattice in the arcs with standard interleaved sextupole chromaticity correction. The chromaticity correction in the HER interaction region is made using "beta-beats" (high single plane betas at the sextupoles) in the nearby arcs. The LER has a 90 degrees per cell lattice in the arcs with a noninterleaved sextupole correction scheme. In the LER interaction region the chromaticity correction is semi-local with the $\mathrm{y}$ correction done in the IR and the $\mathrm{x}$ correction made in the nearby arcs. Both HER and LER lattices as measured are within a few percent of the design. The IP betas are correct horizontally and about $10 \%$ low vertically which helps the beam-beam effect.

The PEP-II RF system uses single cell copper cavities with three HOM absorbers. The HER has four cavities per klystron and the LER two. The cavities are E-beam welded with staged machining and tuning steps. The high power RF windows are ceramic with a stainless steel compression ring and are coated with TiN. The low level RF control system includes feedback loops, comb filters, fast interlocks, network analysis, beam aborts, phase control, high voltage and drive control, and EPICs overall control. Both the HER and LER RF systems are fully functional with nearly full gradient. There are only a few trips per day for the full system. The RF feedback systems are well tuned, reliable, and useful diagnostics.

In PEP-II the HER arc vacuum chambers are extruded copper, the LER arc chambers are aluminum with antechambers, and the straight sections have water cooled cylindrical stainless steel pipes. The HER vacuum chambers are made to absorb over $100 \mathrm{~W} / \mathrm{cm}$ of synchrotron radiation and pumped using distributed ion pumps. The synchrotron radiation in the LER is absorbed in Glidcop (grooved) photon stops located in the pumping chamber antechamber. The beam results show that the HER pressure rise with current is within a factor of two of the desired value and should scrub quickly. The LER vacuum pressure is decreasing more slowly than desired and is now at 10 nT/A. An investigation is underway to determine if the TiN coating, small leaks, or the antechamber outgassing contributes. More vacuum pumping is being installed and the small leaks are being fixed. At above $600 \mathrm{~mA}$ in the LER there is evidence for electron multipacting which depends on the bunch current and the number of bunches resulting in pressure increases.

The existing $50 \mathrm{GeV}$ SLAC linac as modified for the SLC with damping rings is used for PEP-II injection. Intensities per pulse can range up to $4 \times 10^{10}$. The injection systems are working well often with $100 \%$ injection efficiency. The e+ beam has a modest transverse mismatch which is under study.

All beams are stable with bunch-by-bunch feedbacks. Without feedback the LER is stable longitudinally to about $330 \mathrm{~mA}$ and the HER to about $500 \mathrm{~mA}$, agreeing with predictions. Transversely, the LER instability threshold is at least $100 \mathrm{~mA}$, again near the prediction. However, the HER transverse threshold is about 10 to 20 $\mathrm{mA}$ which is much lower that predicted. The data do not point to ions nor resonances cleanly although there is some agreement with each. Without transverse feedback very short trains in either ring are unstable at low currents with the tail of the train oscillating. In the HER, different mode numbers occur at different bunch currents.

In PEP-II the LER and HER beams are collided headon requiring horizontal separation near the interaction point using strong dipoles. These first dipoles and quadrupoles are permanent magnets with $10^{-4}$ field 
tolerances and off-axis beams. The next several magnets are special iron septum quadrupoles to complete the doublet focusing. The compensation of the BABAR solenoid is done with six strong skew quadrupoles on each side of the interaction region per ring.

PEP-II has had collisions 20 days so far with several different bunch trains. Position monitors $72 \mathrm{~cm}$ from the IP are very useful in bringing the beams into collision. The bunch timing is measured to a few ps and held constant with RF phase feedback. Beam-beam scans are done magnetically in both planes. A vertical scan is shown in Fig. 5. Raising the HER bunch charge above $0.4 \mathrm{~mA}$ reduces the LER bunch lifetime. Raising the LER bunch charge even to $3 \mathrm{~mA}$ has not yet hurt the HER bunch lifetime but does reduce its own lifetime. The optimum ratio of bunch charges is near $0.35 \mathrm{~mA}$ HER to about 1.3 $\mathrm{mA}$ LER which is about the design. No significant parasitic collision effects have been seen. The multi-bunch luminosity scales correctly from single bunch luminosity. The luminosity measured over several days is shown in Fig. 6 and the luminosity versus current product in Fig. 7. The specific luminosity is about $60 \%$ of the design. The best luminosity has been achieved with 786 bunches, spaced every four RF buckets. The achieved beam-beam tune shifts are about 0.032 horizontally and 0.013-0.017 vertically, with the horizontal e+ as the only operational limit so far. There is an IP orbit drift associated with air temperature and the HER beam current.

The backgrounds at the IP for the HER now match the new simulations but are a factor of 5 to 7 larger than the original design. The backgrounds for the LER are 10 to 20 times the design. Twelve collimators are being installed.

\section{REFERENCES}

[1] "KEKB B-Factory Design Report", KEK Report 95-7, (1995).

[2] "Design Report on PF Injector Linac Upgrade for KEKB", KEK Report 95-18, (1996).

[3] K. Oide, "Commissioning Results from KEKB," PAC99 proceedings, March 1999.

[4] K. Egawa, etal, "Modeling of the KEKB Colliding Rings," PAC99 proceedings, March 1999.

[5] T. Tajima, "Superconducting Cavity System for KEKB," PAC99 proceedings, March 1999.

[6] M. Tobiyama and E. Kikutani, "Commissioning of the KEKB Bunch Feedback Systems," PAC99 Proceedings, March 1999.

[7] "PEP-II Conceptual Design Report", SLAC Report 418, LBL-PUB5379, June (1993).

[8] M. Sullivan, "Beam-Beam Collisions at PEP-II," PAC99 proceedings, March 1999.

[9] P. Corredoura et al., "Low-level RF System for the PEP-II BFactory", PAC95, p. 2672 (1995) and PAC99 proceedings, March 1999.

[10] M. Zisman, "Commissioning Results of the PEP-II LER," PAC99 proceedings, March 1999.

[11] J. Seeman, "KEKB and PEP-II B Factories," Proceedings of Flavor Physics Conference, Nuc. Phys. B 59 (1997), p. 51.
[12] J. Fox, "Multi-Bunch Instability Diagnostics via Digital Feedback Systems," PAC99 proceedings, March 1999.

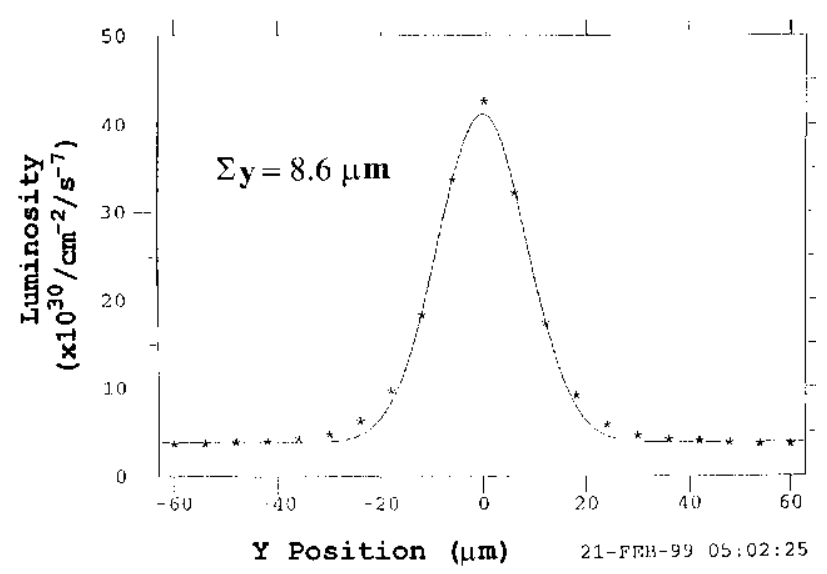

Figure 5: PEP-II vertical beam scan showing luminosity vs beam position. The width gives the combined beam size. The design $\Sigma y$ is 6.7 microns.

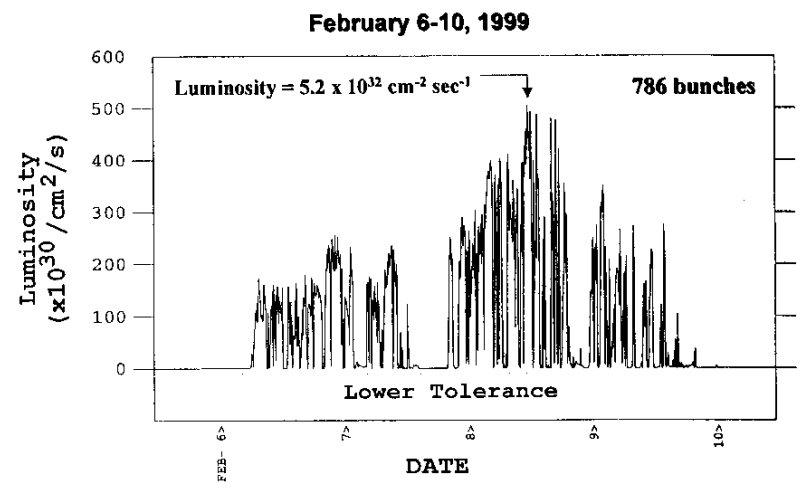

Figure 6: PEP-II Luminosity measured over 72 hours in February 1999 with an average of $2 \times 10 * * 32 / \mathrm{cm}^{* *} 2 / \mathrm{s}$.

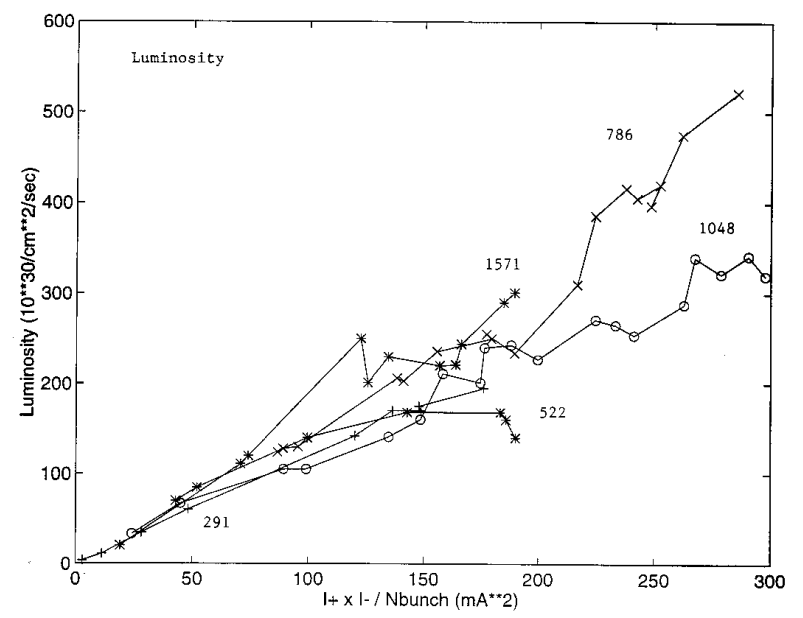

Figure 7: PEP-II measured luminosity versus the product of the beam currents divided by the number of bunches (indicated). At low currents the specific luminosities are nearly the same but at high currents some spot size enlargements occur. 